\title{
Discours
}

Revue de linguistique, psycholinguistique et informatique. A journal of linguistics, psycholinguistics and computational linguistics

$2 \mid 2008$

Varia

\section{Les emplois corrélatifs de parfois, quelquefois et des} fois

\section{Anne-Marie Guiraud}

\section{OpenEdition}

\section{Journals}

Édition électronique

URL : https://journals.openedition.org/discours/3062

DOI : 10.4000/discours.3062

ISSN : 1963-1723

Éditeur :

Laboratoire LATTICE, Presses universitaires de Caen

\section{Référence électronique}

Anne-Marie Guiraud, « Les emplois corrélatifs de parfois, quelquefois et des fois », Discours [En ligne], 2 | 2008, mis en ligne le 11 octobre 2017, consulté le 05 juillet 2022. URL : http://

journals.openedition.org/discours/3062 ; DOI : https://doi.org/10.4000/discours.3062

Ce document a été généré automatiquement le 5 juillet 2022.

\section{(c) (i) () $\Theta$}

Creative Commons - Attribution - Pas d'Utilisation Commerciale - Pas de Modification 4.0 International - CC BY-NC-ND 4.0

https://creativecommons.org/licenses/by-nc-nd/4.0/ 


\title{
Les emplois corrélatifs de parfois, quelquefois et des fois
}

\author{
Anne-Marie Guiraud
}

1 Comme le montre l'extrait suivant tiré d'un corpus littéraire, l'adverbe parfois permet d'exprimer, en français actuel, l'alternance de plusieurs situations à l'intérieur d'une même période temporelle :

[1] Où était-il allé pendant ce temps-là ? Parfois il était retourné à Madagascar, parfois il avait revécu un épisode de Guy l'Éclair ou de Mandrake, ses héros préférés, parfois il avait simplement re-fait un repas ou re-vu un film plus ou moins fragmentairement. (R. Queneau, Le dimanche de la vie, 1952)

Dans [1], la présence de parfois permet d'évoquer trois situations en relation avec l'interrogation précédente. La phrase, composée de propositions indépendantes, sans connecteur, trouve son unité dans le déroulement aléatoire de trois procès à l'intérieur du même intervalle temporel, "pendant ce temps-là ». Des emplois similaires sont relevés avec les formes quelquefois et des fois. Issues du $\mathrm{N}$ fois, elles expriment une fréquence de réalisation plutôt faible; lorsqu'elles sont corrélées, elles cumulent les fréquences de chacun des segments dans un seul intervalle temporel.

Parfois, quelquefois, des fois ne sont pas à proprement parler des « corrélateurs » (cf. infra S. Allaire, M. Charolles, C. Schnedecker), mais leur répétition dans le discours forme écho, et incite le lecteur à comparer les éléments qui les accompagnent. L'effet produit a une conséquence sur la compréhension et l'interprétation de l'énoncé et contribue à configurer une unité textuelle de rang supérieur à l'unité syntaxique. Les éléments corrélés s'opposent ou se complètent. Ce type d'emploi fait-il évoluer les propriétés sémantiques et syntaxiques des formes simples vers des fonctions de corrélateurs?

4 Pour tenter de répondre à cette question, nous définissons dans un premier temps les propriétés sémantiques et syntaxiques des formes simples, puis nous proposons une définition de la corrélation adaptée aux emplois corrélés de parfois, quelquefois, des fois. Nous cherchons ensuite, à partir d'un corpus, à identifier les propriétés les plus 
saillantes de l'emploi des trois formes en corrélation, pour les comparer à celles des formes simples.

\section{Caractéristiques sémantiques et syntaxiques de parfois, quelquefois, des fois, en emploi isolé}

\subsection{Le $\mathbf{N}$ fois : de l'itération à la fréquence}

Les trois formes que nous étudions sont composées à partir du $\mathrm{N}$ fois. Fois vient du latin vices, forme déponente du mot féminin vicis (cas génitif; il n'y a pas de nominatif). Rey et al. (1999 : 1450) précise que la racine indo-européenne est ' weik, qui signifie « céder ». La première signification de vices, mentionnée dans Gaffiot et Flobert $(2001: 806)$ est : " tour ", " succession » qui est à l'origine de l'expression vice versa. Le sens évolue vers la notion de remplacement entre deux personnes, et suggère une différence de hiérarchie entre l'élément d'origine et son remplaçant; en français, il a donné, entre autres, le mot vice qui compose les mots vice-roi, vice-président, etc. Le mot latin signifie aussi «alternatives de la destinée »; le mot dérivé vicissitudo a donné le mot français vicissitude, d'abord au singulier, puis au pluriel, qui dénote l'idée de hasard avec une connotation négative. En latin impérial, le mot vices prend le sens de " tour », «fois »; l'expression latine vice quadam introduit un épisode narratif, comme "une fois » en français. Le nombre d'occurrences d'un même fait n'est plus limité à deux.

Le $\mathrm{N}$ fois dénote les deux propriétés ontologiques du temps et de la quantité; il fixe un repère temporel pour la localisation d'un événement, sans en préciser la chronologie ni la durée. L'événement est considéré du point de vue de son accomplissement (les étapes de son déroulement n'étant pas évoquées) et de sa reproductibilité1.

7 Le $\mathrm{N}$ fois, itératif, permet de compter les événements de la série par prélèvement aléatoire (une fois, $n$ fois, une fois ou deux), ou en séquence chronologique (les trois premières fois, la énième fois), ou encore à partir d'un repère (la fois suivante, les cinq fois précédentes).

8 Avec les adverbiaux parfois, quelquefois, des fois, le sens de fois évolue vers la fréquence, c'est-à-dire vers la reproduction régulière d'un même phénomène temporel dans un intervalle de temps donné. Le passage de l'itération à la fréquence nécessite un processus de transformation dont les principales caractéristiques ont été décrites par De Swart (1988) :

- Dans l'itération, le comptage s'effectue en valeur absolue. Avec un adverbe de fréquence, il est relatif : le nombre calculé est mis en relation avec une période donnée ; par exemple : la plupart des fois apprécie une quantité par rapport à la totalité des fois possibles dans l'intervalle temporel.

- Les adverbes d'itération s'utilisent presque exclusivement dans des phrases au passé simple, ou au passé composé, éventuellement au futur ; si la phrase est au présent ou à l'imparfait, elle ne peut avoir qu'une lecture d'habitude, par exemple : ?'Paul vient deux fois. Les adverbes de fréquence n'ont pas de restriction sur les temps verbaux, par exemple : Paul vient parfois.

- A l'intérieur de l'intervalle temporel, la distribution des événements décrite par les adverbes de fréquence est régulière et doit le rester si l'action continue à se dérouler dans le présent; par exemple : Marie joue du piano deux fois par jour suppose que l'action de jouer du piano s'est opérée et s'opère encore tous les jours à deux reprises. Se référant à Stump (1981), De Swart (1988 : 150) précise : 
In Stump (1981) the regular distribution of events over the period which is the temporal projection of the sentence is considered to be a characteristic property of frequency adverbs.

Les adverbiaux temporels de fréquence remplissent l'ensemble des conditions cidessus. Ils n'acceptent pas un découpage en sous périodes de temps (*Parfois par an, *Quelquefois par an), parce qu'ils s'appuient sur un intervalle temporel supposé accessible dans le contexte. Les traits sémantiques de la fréquence peuvent être identifiés comme suit :

- Prise en compte d'un intervalle temporel implicite ou explicite

- Calcul d'une proportion d'occasions réalisées par rapport à un total théorique dans l'intervalle temporel

- Répartition régulière des événements dans l'intervalle temporel.

11 Nous avons établi en figure 1 une échelle de fréquence qui classe les adverbiaux temporels en fonction du pourcentage d'occasions réalisées qu'ils dénotent :

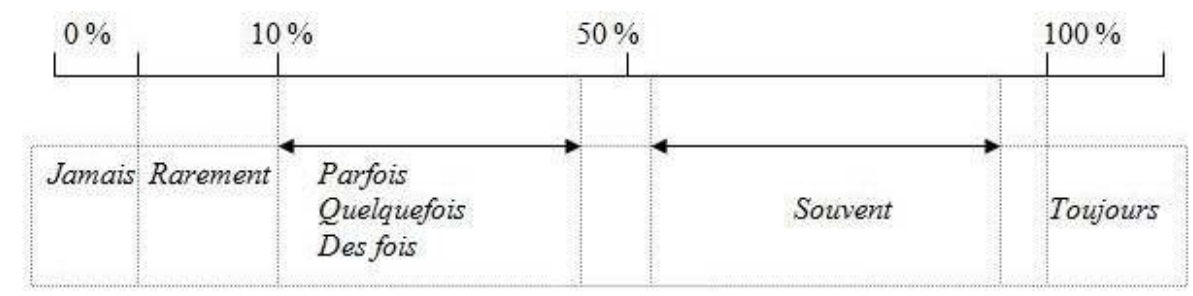

Figure 1 : échelle de répartition des adverbes de fréquence en fonction de la proportion d'occasions réalisées par rapport à un total théorique

Parfois, quelquefois et des fois expriment une proportion sensiblement comparable, inférieure à la moitié des occurrences, moins négligeable que rarement.

\subsection{Parfois}

Parfois est issu de la soudure de par et de fois ; la forme soudée est attestée une fois à la fin du XIII ${ }^{\mathrm{e}}$ siècle et de nouveau au XV siècle, d'après Rey et al. (1999: 2575). La préposition par, employée sans déterminant, exprime l'idée d'une «répartition discontinue à l'intérieur d'une totalité, d'un processus global » selon le TLFi ${ }^{2}$. Avec le N fois, le processus concerné par la répartition est temporel. Berthonneau (1988: 507) note :

Parfois, composé d'une préposition et de fois, et non avec un déterminant, a été rattaché au paradigme de par donnant une validation discontinue du procès. C'est de par qu'est tirée la discontinuité impliquée par cet adverbe.

Berthonneau (ibid : 302-303) définit ainsi la «validation discontinue » et l'applique à l'adverbe parfois :

Quel que soit le nom [qu'il soit temporel ou non], l'interprétation est de type « Il y a des $\mathrm{N}$ (moments, endroits) où $\mathrm{P}$ est vraie et des $\mathrm{N}$ où $\mathrm{P}$ n'est pas vraie » [...] L'adverbe parfois trouve enfin ici son paradigme naturel. Il correspond bien au sens du complément « Il $\mathrm{y}$ a des fois où $\mathrm{P}$ et des fois où non-P » 
Les traits sémantiques de parfois, hérités de ses composants, sont les suivants :

- Prise en compte d'un intervalle temporel implicite ou explicite

- Répartition discontinue des occurrences, comparées à un total théorique

- Proportion d'occasions réalisées voisine de la moitié des occasions possibles.

La construction avec déterminant, par des fois, est attestée au XVI siècle, avec un sens proche et elle est encore signalée comme régionalisme au XIX ${ }^{e}$ siècle par le TLFi. L'emploi en proposition hypothétique, si parfois, perd le trait temporel et ne conserve que la valeur d'hypothèse, avec une nuance de doute :

[2] Un homme ouvre la marche avec un crucifix voilé de deuil. Tu vois leurs pieds nus?

- Ils se font mal?

- Qui sait si parfois du verre n'a pas été jeté à dessein? (R. Sabatier, Les Noisettes sauvages, 1974)

Un glissement comparable du temporel à l'hypothétique est relevé par Grevisse (1993: 589) qui signale sous forme de régionalisme l'usage de parfois dans une interrogation globale, pour la rendre plus prudente avec souvent un verbe au conditionnel. L'adverbe prend une valeur de modalisation (doute) :

[3] Auriez-vous parfois fait cela? (Exemple de Grevisse)

\subsection{Quelquefois}

Quelquefois provient de la soudure de quelques et de fois ; la forme soudée est attestée en 1539, selon Rey et al. (1999: 3038), mais on la trouve en fait dès les Mémoires de Commynes, en $1490^{3}$. Quelques, déterminant, employé au pluriel, désigne un nombre indéterminé, mais faible. Le prélèvement des occurrences est aléatoire. Les traits sémantiques de l'intervalle temporel et de la régularité n'apparaissent pas dans les composants de quelquefois ; c'est sans doute ce qui justifie la persistance de la forme non soudée, quelques fois, à valeur itérative.

La soudure des deux mots a fait disparaître le $s$ final de quelques. Le mot s'emploie d'abord avec les deux significations de quelques fois et de une fois. Au XVII ${ }^{\mathrm{e}}$ siècle, le sens évolue vers la fréquence par mise en relation du nombre représenté par quelques avec une norme en vigueur dans une période donnée. Le mot est comparé à parfois et à d'autres adverbiaux temporels de fréquence :

[4] Il aime quelquefois sans qu'il le sache bien,

Et croit aimer aussi parfois qu'il n'en est rien. (Molière, Le Misanthrope, 1667)

Les traits sémantiques de quelquefois, hérités de ses composants, sont les suivants :

- Intervalle temporel récupérable en contexte

- Répartition aléatoire des prélèvements, comparés à un total théorique

- Proportion d'occasions réalisées faible

- Le prélèvement apparaît nettement moins régulier qu'avec parfois.

Si quelquefois réduit les possibilités de réalisation de l'éventualité exprimée ; quelquefois que, en langage familier, est relevé par Rey et al. (1999 : 3038), au sens de «si jamais par hasard ». Un glissement du temporel à l'hypothétique, comparable à celui de si parfois, est constaté par Grevisse (1993: 589) qui signale l'usage de quelquefois dans une 
interrogation globale, pour la rendre plus dubitative, avec éventuellement un verbe au conditionnel :

[5] Vous n'auriez pas vu quelquefois mon perroquet? (Exemple de Grevisse)

\subsection{Des fois}

21 La forme des fois est plus familière et plus tardive que les deux formes précédentes. L'article indéfini, toujours pluriel, indique un prélèvement indifférencié et indéterminé d'occurrences dans un ensemble. La forme est relevée dans Frantext au XIX ${ }^{\mathrm{e}}$ siècle, au sens de plusieurs fois.

Les traits sémantiques de des fois, hérités de ses composants, sont les suivants :

- Intervalle temporel récupérable en contexte

- Répartition aléatoire des prélèvements, comparés à un total théorique

- Nombre indéterminé d'occurrences, inférieur à la moitié du total théorique

- Le prélèvement apparaît nettement moins régulier qu'avec parfois.

La forme bien des fois exprime une fréquence plus élevée, équivalant à «souvent ». Des fois et des fois, au $\mathrm{xx}^{\mathrm{e}}$ siècle, apporte une nuance d'excès. Si des fois, des fois que, hypothétiques, signifient « si par hasard »; le trait temporel disparaît. Grevisse (1993 : 589) note la présence de des fois que dans une interrogation oratoire, avec une nuance ironique :

[6] C'est-y des fois que ces gaillards-là vont avoir le toupet de nous laisser ici et nous lâcher l'train sous le nez?» (G. Courteline, Le Train de 8 heures 47, 1888. Cité par Grevisse)

Des emplois purement exclamatifs de des fois apparaissent au $\mathrm{XIX}^{\mathrm{e}}$ siècle dans les controverses. L'interjection Non mais des fois récuse les propos précédents, elle est proche de: Non mais attends, introducteur d'une réfutation, étudié par KerbratOrecchioni (2005: 54), qui « vise à faire cesser un type de discours envisagé du point de vue de son contenu ». Berthonneau (1988: 507) remarque que la présence de non mais dans l'interjection est indispensable; c'est elle en effet qui porte la controverse. L'interjection ne porte plus de trait temporel, elle exprime une relation interpersonnelle :

[7] STEPHANO : Je me couronne roi de l'île.

TRINCULO : Ouais ! Pourquoi toi ? Je suis le premier qui y ai pensé, à cette couronne.

STEPHANO : Dis donc, Trinculo! Non mais des fois! Tu ne t'es pas regardé ! Qu'est-ce qu'il faut à un roi ? De la prestance. Et moi, c'est pas pour dire, j'ai de la prestance. (A. Césaire, Une tempête, 1969)

L'affaiblissement sémantique, au profit de l'expression de l'attitude du locuteur, rappelle le phénomène de « subjectification » décrit par Traugott (1995:32) :

[Subjectification is specifically a] GRADIENT phenomenon, whereby forms and constructions that at first express primarily concrete, lexical, and objective meanings come through repeated use in local syntactic contexts to serve INCREASINGLY abstract, pragmatic, interpersonal, and speaker-based functions. même temps que le trait temporel disparaît. L'emploi exclamatif correspondrait au 
stade suivant d'intersubjectification décrit par Traugott (2003) comme "the explicit expression of the speaker/writer's attention to the « self » of addressee/reader ».

\subsection{Comparaison de parfois, quelquefois, des fois}

27 Au plan sémantique, les trois formes présentent les différences suivantes :

- Parfois est la forme qui porte le plus de traits sémantiques de la fréquence : répartition discontinue à l'intérieur d'un intervalle temporel implicite ou explicite, proportion prélevée proche de la moitié;

- Quelquefois et des fois dénotent une proportion plus faible; l'intervalle temporel est récupérable en contexte ; la répartition est présentée comme nettement moins régulière que pour parfois.

Avec si ou le conditionnel, le trait temporel disparaît, remplacé par une valeur hypothétique, complétée de valeurs modales (doute, ironie). Une construction de même sens est possible avec que pour des fois (des fois que), plus rarement pour quelquefois (quelquefois que), mais n'apparaît pas avec parfois (*parfois que).

La désémantisation s'accentue avec des fois dans les formes exclamatives : non mais des fois évolue vers l'intersubjectification.

$\mathrm{Au}$ plan syntaxique, les trois formes que nous étudions peuvent avoir un emploi extraprédicatif, selon la terminologie de Guimier (1996: 72) :

Si l'adverbe postposé est incident au verbe, et à ce titre élément constitutif du prédicat (intra-prédicatif), l'adverbe antéposé et détaché intonativement est incident à la relation sujet-prédicat; il n'est pas, pour cette raison, élément constitutif du prédicat. Un tel adverbe sera dit extra-prédicatif.

L'emploi extraprédicatif est illustré en [8], [9] et [10] ci-dessous ; les adverbiaux sont souvent en tête de phrase, mais restent très mobiles, comme en [9] où quelquefois est placé en incise après un autre adverbial :

[8] Parfois nous doutions qu'un terrain alluvionnaire pût se combler en si peu d'années, mais ma mère nous rassurait. (M. Duras, Cahiers de la guerre et autres textes, 2006)

[9] Jamais en tout cas, ils ne me demandent un sou. Jamais on ne manque de rien. Au contraire, quelquefois, on a trop de quelque chose. (F. Dorin, Les Vendanges tardives, 1997)

[10] Petit Michel est assis sur la cuvette, dos appuyé au mur. Il pense à sa mère. Lui, il sait qu'elle n'est pas vraiment malade. Des fois, quand il est seul avec elle, qu'elle cire les meubles, elle se retourne et lui sourit. (V. Thèrame, Bastienne, 1985)

Parfois et quelquefois ont souvent un emploi intraprédicatif, moins mobile dans la phrase ; cet emploi est plus rare pour des fois. 


\section{Caractéristiques des trois formes dans les emplois corrélatifs}

\subsection{Propriétés des corrélatifs}

trois catégories : structurales, lexicales, sémantico-pragmatiques. Il propose de traiter les relations de dépendances en termes de degré; les plus hauts degrés de dépendance se situent au niveau des relations syntaxiques; à un degré moindre, les corrélateurs (plus ... plus, ou non seulement ... mais encore) impliquent une dépendance entre les phrases, annoncée dès le premier segment par l'occurrence d'une expression lexicale ; à un degré encore plus faible, des liens de dépendance peuvent s'établir au niveau structural ou lexical (parallélisme de construction, marques lexicales); le niveau le plus bas concerne des liens de dépendance au niveau lexical. Dans cette échelle, parfois, quelquefois, des fois peuvent être considérés comme des corrélateurs "faibles", correspondant à des degrés de dépendance plus ou moins lâches. Ainsi, les moyens structuraux et lexicaux sont mis en œuvre dans l'exemple [1] que nous reprenons cidessous :

[1] Où était-il allé pendant ce temps-là ? Parfois il était retourné à Madagascar, parfois il avait revécu un épisode de Guy l'Éclair ou de Mandrake, ses héros préférés, parfois il avait simplement re-fait un repas ou re-vu un film plus ou moins fragmentairement. (R. Queneau, Le dimanche de la vie, 1952)

Le premier segment n'implique pas la présence d'autres segments similaires; le texte pourrait être énoncé comme suit :

[1'] Où était-il allé pendant ce temps-là ? Parfois il était retourné à Madagascar. En effet, il en conservait un excellent souvenir ...

C'est l'effet de parallélisme qui s'établit progressivement à la lecture du deuxième, puis du troisième segment qui construit l'unité de l'énoncé: les trois segments sont composés de parfois suivi du pronom sujet $i l$, d'un verbe au plus que parfait, et d'un 
groupe complément du verbe. Les similitudes lexicales sont marquées par le préfixe rede chaque verbe, détaché par un tiret dans le troisième segment pour accentuer l'effet de réitération. L'intonation met en valeur la similitude du début et l'allongement progressif de la fin de chaque segment. Le lecteur regroupe mentalement les trois segments et les interprète comme trois possibilités concurrentes à l'intérieur du même intervalle temporel.

Les relations sémantico-pragmatiques (connecteurs, reprise anaphorique, opposition entre une assertion et sa négation) sont mises en œuvre dans l'exemple ci-dessous. Les verbes aider et détruire impliquent des actions différentes mais pas exactement contraires ; l'opposition est marquée par le connecteur et et par le complément d'objet repris anaphoriquement :

[11] L'expérience des remèdes et le bon sens ont établi la médecine pratique dans toute la terre : elle est partout un art conjectural, qui aide quelquefois la nature, et quelquefois la détruit. (Voltaire, Essay sur l'histoire générale et sur les moeurs et sur l'esprit des nations, 1756)

Au niveau du discours, la corrélation forme une unité textuelle indépendante de la syntaxe, et peut dépasser les limites de la phrase.

Pour étudier la structure des phrases corrélatives, nous suivrons les analyses proposées par Schnedecker (2006: 199). Selon elle, l'observation de la corrélation doit tenir compte d'un contexte élargi pour être analysée dans sa totalité. Bien que les formes étudiées par cet auteur (les pronoms l'un ... l'autre) soient éloignées des adverbiaux temporels, deux caractéristiques de la corrélation peuvent servir à notre étude: Schnedecker (2006: 17) note que les éléments qui rapprochent les deux pronoms l'un l'autre sont, outre leurs ressemblances formelles et leurs emplois couplés, des liens au niveau sémantique (énumération ou opposition); au plan discursif, ils peuvent se situer dans des phrases graphiques distinctes. La recherche d'une zone élargie autour des corrélateurs permet d'organiser en trois parties les éléments de phrase à observer :

- La zone d'antécédence (ZA), à gauche de la corrélation, introduit la structure d'accueil. Il ne s'agit pas, avec des adverbiaux temporels, de rechercher les sources des pronoms anaphoriques, mais des indices qui permettraient d'identifier l'introduction d'une nouvelle séquence de discours. En [12], la ZA est la proposition subordonnée («quand il veut »), les segments corrélés reprennent le complément de temps :

[12] Il se lève quand il veut, des fois à quatre heures, des fois à cinq : il est son maître. (J. Renard, Journal, 1910)

- La zone de corrélation $(\mathrm{ZC})$ contient les éléments corrélés

- La zone post-corrélative (ZPC) indique la fin de la corrélation. Elle marque souvent une rupture dans le discours. En [13], la ZPC est introduite par mais et une ponctuation explicative (deux points), avec changement du temps verbal ; elle porte une conclusion :

[13] Je me parcours alors moi-même, et membre à membre, je m'examine, et quelquefois je marche, et quelquefois je cours avec des jointures flexibles, selon qu'une vigueur animée me conduit : mais qui j'étais, où j'étais, par quelle cause j'étais, je ne le savais pas. (F. de Chateaubriand, Le Paradis perdu [trad.], 1839) 


\subsection{Etude sur corpus}

Le corpus d'emplois de parfois, quelquefois, des fois que nous avons constitué est issu de la base informatisée Frantext ${ }^{4}$. Les résultats obtenus pour parfois et quelquefois étant trop élevés ${ }^{5}$, nous avons sélectionné les occurrences les plus anciennes, les plus variées, et celles qui avaient le plus grand nombre de maillons; ce choix avait pour objet de repérer l'évolution diachronique, les différentes combinaisons possibles, et la taille maximum que pouvait atteindre une corrélation. Pour la forme des fois, nous n'avons obtenu au total que 49 occurrences, après avoir complété la recherche avec des corrélations composées de des fois ... d'autres fois; une construction au singulier, de forme l'une des fois ... l'autre des fois, relevée au $\mathrm{XVI}^{\mathrm{e}}$ siècle, a été écartée comme atypique. Le corpus obtenu est le suivant :

- 108 corrélations avec parfois, à partir du XIX ${ }^{\mathrm{e}}$ siècle

- 112 corrélations avec quelquefois, surtout nombreuses au XVII siècle

- 49 corrélations avec des fois, à partir du XIX ${ }^{\mathrm{e}}$ siècle.

La faible représentation de corrélations au XVIII ${ }^{\mathrm{e}}$ siècle est peut-être due au mode de sélection du corpus.

Nous avons cherché, d'une part à qualifier la zone de corrélation, d'autre part à identifier la zone d'antécédence et la zone post-corrélative à partir des marqueurs d'ouverture ou de fermeture de la corrélation.

\subsubsection{La zone de corrélation}

Les premières apparitions de corrélations avec parfois et quelquefois dans Frantext datent $\mathrm{du} \mathrm{XVI}{ }^{\mathrm{e}}$ siècle. La première apparition d'une corrélation avec des fois date du XIX ${ }^{\mathrm{e}}$ siècle (1851).

Trois principaux schémas de corrélations peuvent être distingués : celles formées avec une seule des formes étudiées, celles où l'un au moins des segments corrélés contient un corrélateur différent et celles contenant deux des formes étudiées.

Le premier schéma correspond à des corrélations de format : parfois ... parfois ... (parfois ...), ou quelquefois ... quelquefois ... (quelquefois ...), ou des fois ... des fois / d'autres fois ... (des fois / d'autres fois). Le tableau 1 montre la répartition de ce schéma par nombre de maillons et par siècle. 


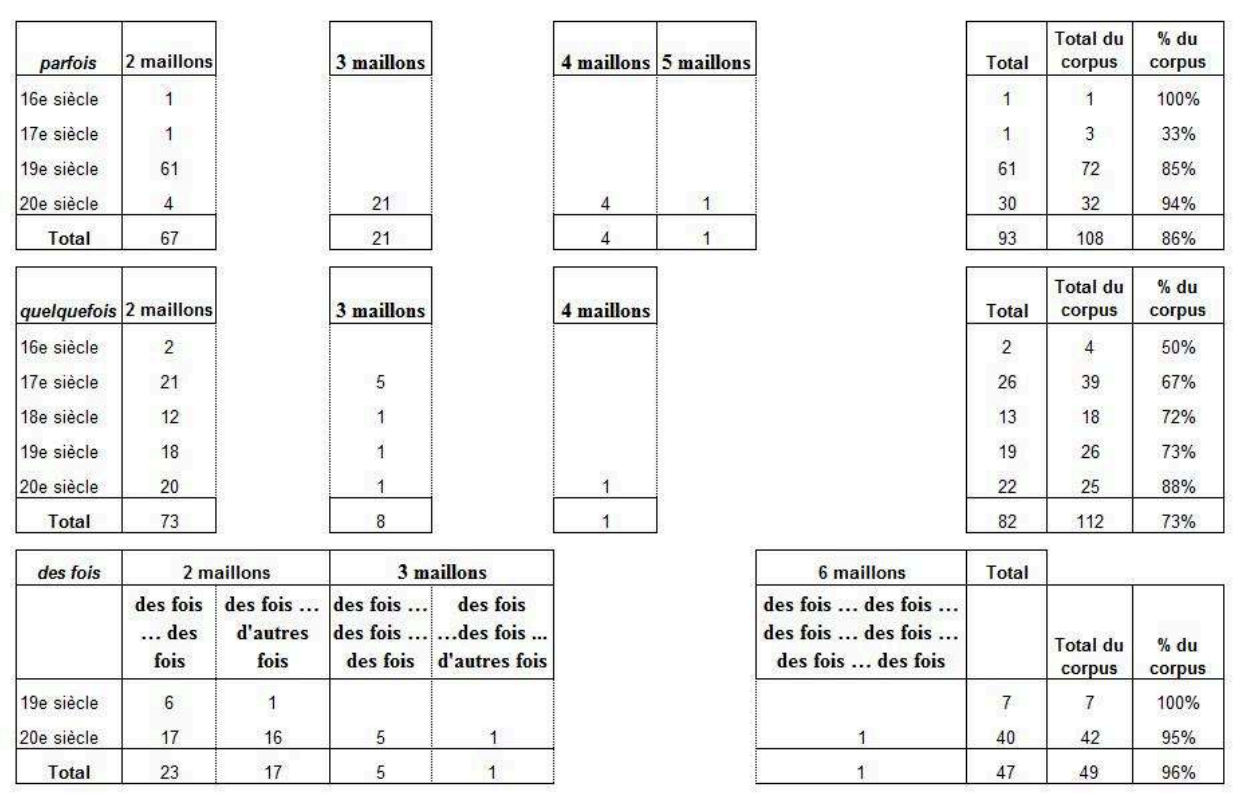

Tableau 1 : corrélations avec une seule forme - Répartition de parfois, quelquefois, des fois par siècles et nombre de maillons

[14] Il s'habille quelquefois, et quelquefois il fait toutes ces choses à demi habillé, et quelquefois n'ayant que sa chemise. (F. Bernier, Abrégé de la philosophie de Gassendi, 1684)

Les corrélations avec des fois se terminent dans $40 \%$ des cas par un segment contenant d'autres fois. La recherche de corrélations à un maillon dans Frantext montre que la situation contraire peut être sous-entendue avec des fois, si l'effet de contraste est suffisamment inféré, comme dans l'exemple ci-dessous. La proportion de réalisations exprimée est inférieure à $50 \%$, l'énoncé sous-entend que la situation inverse se produit le reste du temps :

[15] Elle poursuivit après un intervalle de silence :

- Des fois, quand j'ai fini l'ouvrage et qu'on veut bien, je m'amuse aussi. (V.

Hugo, Les Misérables, 1881)

Le second schéma de corrélation associe la forme parfois, quelquefois, des fois à d'autres marqueurs de corrélation: le corrélateur tantôt, avec d'autres adverbes temporels ou avec d'autres adverbiaux. Le tableau 2 présente les différentes combinaisons relevées. 

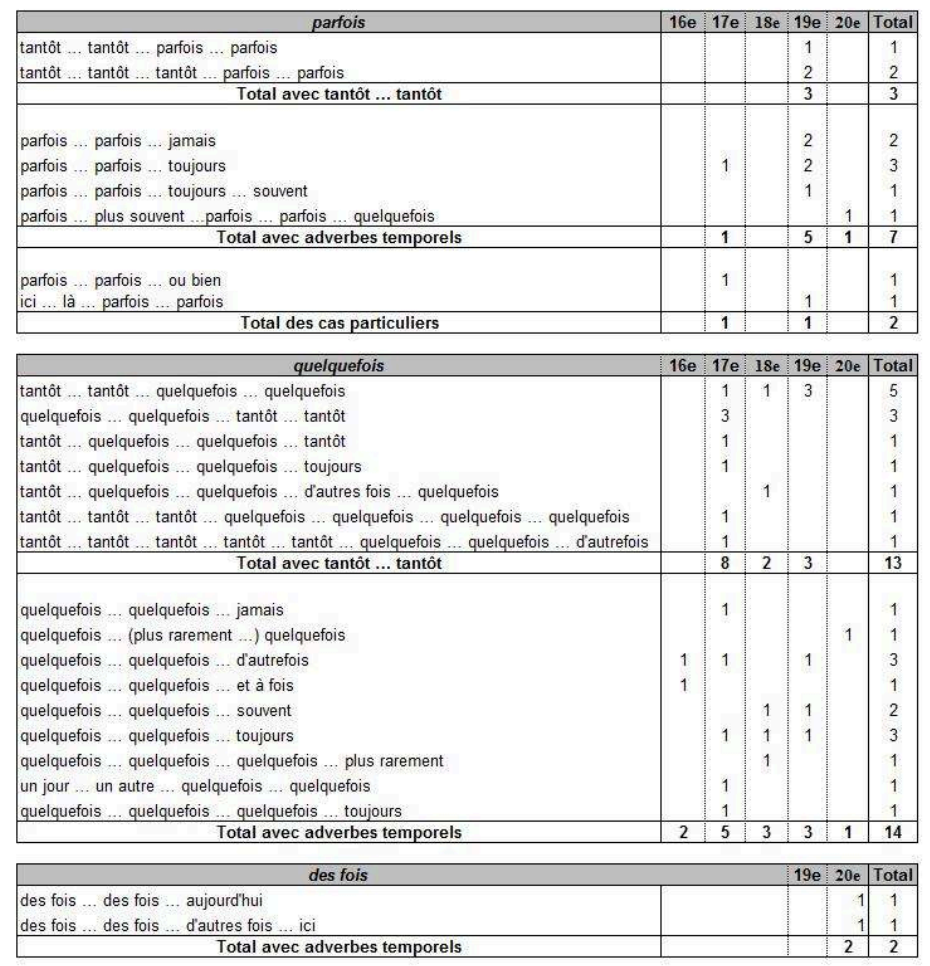

Tableau 2 : corrélations composées avec d'autres corrélateurs et / ou d'autres adverbes temporels Répartition chronologique des différents schémas composés avec parfois, quelquefois, des fois

51 Adverbe temporel à l'origine, tantôt prend dès le $\mathrm{XVI}^{\mathrm{e}}$ siècle, selon le TLFi, des fonctions de corrélateur "fort» pour marquer des «changements consécutifs et qui se répètent». Les associations les plus nombreuses avec tantôt sont relevées dans les corrélations contenant quelquefois aux $\mathrm{XVII}^{\mathrm{e}}$ et $\mathrm{XVIII}{ }^{\mathrm{e}}$ siècles :

[16] Tantost il estimoit la blancheur de son visage, tantost la vive couleur du teint, tantost la vivacité de ses yeux, tantost le corail de sa bouche, tantost l'yvoire de ses dents, quelquefois sa belle taille, quelquefois la delicatesse et blancheur de sa main, et d'autrefois l'avantage qu'elle avoit sur toutes ses compagnes de sçavoir si bien se prevaloir des habits et de tout ce qu'elle mettoit. (H. d'Urfé, L'Astrée, 1627)

Aucune corrélation avec tantôt n'est relevée dans Frantext au $\mathrm{Xx}^{\mathrm{e}}$ siècle.

Dans la corrélation avec parfois ou quelquefois, les autres adverbes de fréquence apportent des points de vue indépendants. En [17], les deux segments avec parfois proposent une alternative; un autre point de vue est décrit avec toujours; une troisième perspective est proposée avec souvent dans le dernier segment:

[17] Il crut pouvoir y réussir en badinant avec l'inconnue à la faveur de cette amabilité française, de cet esprit parfois léger, parfois sérieux, toujours chevaleresque, souvent moqueur qui distinguait les hommes remarquables de l'aristocratie exilée. (H. de Balzac, Les Chouans ou la Bretagne en 1799, 1845)

Dans de nombreuses corrélations, une seule occurrence de parfois et quelquefois est combinée avec un autre adverbe de fréquence ; jamais et toujours couvrent l'ensemble des possibles, souvent comble les possibilités de réalisation non couvertes par parfois et 
quelquefois. En [18], la plus faible partie des occasions est réalisée avec parfois, le complément avec souvent :

[18] Anglarès se dégoûtant aussi vite qu'il s'enthousiasmait le néophyte disparaissait, parfois sans bruit, souvent avec fracas. (R. Queneau, Odile, 1937)

Exceptionnellement, les corrélations avec des fois sont complétées par des adverbes qui réfèrent à la situation d'énonciation : aujourd'hui, ici. Les occasions précédentes sont évoquées par des fois, mais celle dont il sera question dans la suite du récit est introduite par aujourd'hui ou par ici, qui localise un repère spatial autant que temporel :

[19] Il y avait toujours un désavantage. Des fois, c'était l'ombre ; des fois, c'était le voisinage d'une usine; d'autre fois, c'était l'exiguïté du logement ; ici, c'était la proximité du chemin de fer. (G. Roy, Bonheur d'occasion, 1945)

Une corrélation avec parfois, au XVII ${ }^{\mathrm{e}}$ siècle, est complétée par ou bien. Une autre, au XIX siècle, est de forme : Ici ... là ... parfois... parfois. La propriété sémantique de par, qui décrit une validation discontinue du procès dans le temps ou dans l'espace, facilite le rapprochement entre les adverbes spatiaux et parfois. Les deux groupes, spatial et temporel, sont liés par une structure de chiasme au niveau lexical (creuser, élever / haut, profond) et dans la composition des segments (complément, verbe, adverbe / adverbe, complément) :

[20] Cette rivière était tranquille dans le comté de Hartford, à soixante milles de Londres ; le chevalier Middleton vint et la prit ; il amena une brigade de six cents hommes armés de pelles et de pioches, se mit à remuer la terre, la creusant ici, l'élevant là, parfois vingt pieds haut, parfois trente pieds profond, fit des aqueducs de bois en l'air, et çà et là huit cents ponts, de pierre, de brique, de madriers, et un beau matin, la rivière entra dans Londres, qui manquait d'eau. (V. Hugo, L'Homme qui rit, 1869)

Le troisième schéma de corrélation concerne des corrélations où parfois, quelquefois et des fois sont présents. Le tableau 3 présente les différentes combinaisons relevées. 


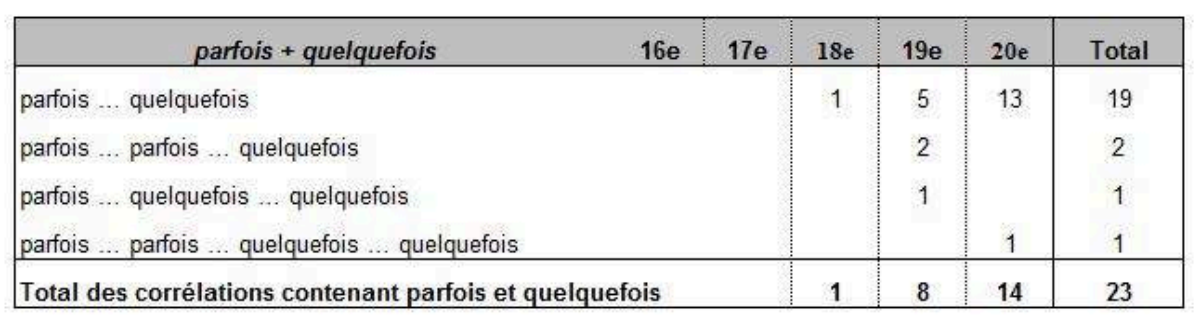

\begin{tabular}{|l|c|c|c|c|c|}
\hline \multicolumn{1}{|c|}{ quelquefois + parfois } & $\mathbf{1 6 e}$ & $\mathbf{1 8 \mathrm { e }}$ & $\mathbf{1 9 \mathrm { e }}$ & $\mathbf{2 0 \mathrm { e }}$ & Total \\
\hline quelquefois ... parfois & 1 & 1 & 2 & 6 & 10 \\
quelquefois ... quelquefois ... parfois & & & & 1 & 1 \\
\hline Total des corrélations contenant quelquefois et parfois & $\mathbf{1}$ & $\mathbf{1}$ & $\mathbf{2}$ & $\mathbf{7}$ & $\mathbf{1 1}$ \\
\hline
\end{tabular}

\begin{tabular}{|cc|c|c|}
\hline parfois + des fois & $19 \mathrm{e}$ & $\mathbf{2 0 e}$ & Total \\
\hline parfois ... parfois .... Des fois & 1 & 1 \\
\hline
\end{tabular}

\begin{tabular}{|c|c|c|c|}
\hline quelquefois + des fois & $19 \mathrm{e}$ & $20 \mathrm{e}$ & Total \\
\hline quelquefois ... des fois ... pour le moment & & 1 & 1 \\
\hline
\end{tabular}

Tableau 3 : corrélations composées de parfois, quelquefois, des fois - Répartition chronologique des différents schémas associant deus des formes étudiées juxtaposent parfois et quelquefois; l'effet obtenu est une séparation en sous-groupes, comme dans [21], où deux segments avec parfois sont suivis de deux segments avec quelquefois ${ }^{6}$. Le premier groupe concerne le discours, le second la posture du personnage, dans le même intervalle temporel :

[21] Il prononçait quelques phrases, parfois brisées de silences, parfois s'enchaînant avec la continuité monotone d'une prière. Quelquefois immobile contre la cheminée, comme une cariatide, quelquefois s'approchant, sans s'interrompre, d'un objet, d'un dessin au mur. (Vercors, Le Silence de la mer, 1942)

59 proches sans être égales, et l'auteur joue sur cette ambiguïté :

[22] Écoutez à présent. - Dans ma raison qui tremble,

Parfois l'une après l'autre et quelquefois ensemble,

Trois voix, trois grandes voix murmurent. (V. Hugo, Les Rayons et les ombres, 1840)

Des fois s'associe rarement avec parfois ou quelquefois. En [23], les deux segments corrélés avec parfois s'opposent l'un à l'autre. Le troisième segment avec des fois présente une autre possibilité, mise en valeur par une ponctuation forte :

[23] Parfois je m'imagine que je ne suis plus le même, que je sors réellement de ma peau, parfois non. Et des fois encore, je doute, c'est le plus dur... je me joue le guignol tout seul, pour moi seul. (G. Bernanos, Monsieur Ouine, 1943)

61 En [24], l'alternance de quelquefois et de des fois suggère des règles vagues. Pour le moment, dans le troisième maillon, réfère à la situation d'énonciation et relègue dans le 
passé les deux premiers segments, selon un procédé que nous avons déjà relevé dans les corrélations avec des fois :

[24] Il y a quatre divisions, à cette heure, au corps d'armée, répond Cocon. Ça change : quelquefois c'est trois, des fois, c'est cinq. Pour le moment, c'est quatre. (H. Barbusse, le Feu, 1916)

Le tableau 4 présente le nombre de maillons des corrélations tous marqueurs confondus. Le schéma privilégié est binaire: deux possibilités s'opposent ou se complètent dans une formulation brève.

\begin{tabular}{|c|c|c|c|c|}
\hline parfois & 2 maillons & 3 maillons & 4 maillons & 5 maillons \\
\hline $16 \mathrm{e}$ siècle & 1 & & & \\
$17 \mathrm{e}$ siècle & 1 & 2 & & \\
$19 \mathrm{e}$ siècle & 61 & 6 & 3 & 2 \\
$20 \mathrm{e}$ siècle & 4 & 22 & 4 & 2 \\
\hline Total & 67 & 30 & 7 & 4 \\
\hline
\end{tabular}

\begin{tabular}{|c|}
\hline Total \\
\hline 1 \\
3 \\
72 \\
32 \\
\hline 108 \\
\hline
\end{tabular}

\begin{tabular}{|l|c|c|c|c|}
\hline quelquefois & 2 maillons & 3 maillons & 4 maillons & 5 maillons \\
\hline $16 \mathrm{e}$ siècle & 2 & 2 & & \\
$17 \mathrm{e}$ siècle & 21 & 8 & 8 & \\
$18 \mathrm{e}$ siècle & 12 & 3 & 2 & 1 \\
$19 \mathrm{e}$ siècle & 18 & 5 & 3 & \\
$20 \mathrm{e}$ siècle & 20 & 3 & 2 & \\
\hline Total & $\mathbf{7 3}$ & $\mathbf{2 1}$ & $\mathbf{1 5}$ & $\mathbf{1}$ \\
\hline
\end{tabular}

\begin{tabular}{|c|c|c|}
\hline $\mathbf{7}$ maillons & $\mathbf{8}$ maillons & Total \\
\hline & & 4 \\
& 1 & 39 \\
& & 18 \\
& & 26 \\
& & 25 \\
\hline 1 & 1 & 112 \\
\hline
\end{tabular}

\begin{tabular}{|c|c|c|c|}
\hline des fois & 2 maillons & 3 maillons & 4 maillons \\
\hline $19 \mathrm{e}$ siècle & 7 & & \\
$20 \mathrm{e}$ siècle & 33 & 7 & 1 \\
\hline Total & 40 & 7 & 1 \\
\hline
\end{tabular}

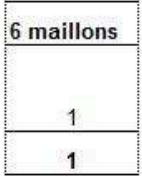

\begin{tabular}{|c|}
\hline Total \\
\hline 7 \\
42 \\
\hline 49 \\
\hline
\end{tabular}

Tableau 4 : répartition de parfois, quelquefois, des fois par siècle et nombre total de maillons de la corrélation

Les relations de sens portées par les segments sont principalement l'opposition, ou l'addition dans une série. Une relation plus marginale est l'imbrication.

\begin{tabular}{|c|c|c|c|}
\hline & Parfois & Quelquefois & Des fois \\
\hline \multicolumn{4}{|c|}{ Volumes concernés par les relations d'opposition } \\
\hline Total des relations d'opposition & 48 & 53 & 24 \\
\hline Relations d'opposition où plusieurs segments sont soutenus par des connecteurs & 25 & 27 & 10 \\
\hline Relations d'opposition clôturées par des connecteurs & 2 & 3 & \\
\hline Relations d'opposition regroupées en sous-ensembles par des connecteurs & & 3 & \\
\hline \multicolumn{4}{|c|}{ Nombre de maillons des relations d'opposition } \\
\hline Deux maillons & 40 & 36 & 24 \\
\hline Trois maillons & 5 & 7 & \\
\hline Quatre maillons & 3 & 9 & \\
\hline Sept maillons & & 1 & \\
\hline \multicolumn{4}{|c|}{ Nombre de corrélations en relations d'oppositiondes utilisant des connecteurs } \\
\hline ( & 2 & 1 & \\
\hline Et & 17 & 21 & 4 \\
\hline Et ... aussi & 4 & & \\
\hline Mais & & 3 & 2 \\
\hline Mais ... aussi & & 3 & 1 \\
\hline Aussi & 3 & 5 & 1 \\
\hline Puis & & & 2 \\
\hline Au contraire & 1 & & \\
\hline
\end{tabular}

Tableau 5 : caractéristiques des relations d'opposition 

deux segments contraires couvrent toutes les possibilités :

[25] [...] les gens allaient lui demander d'arranger toutes leurs petites affaires quand ça ne marchait pas comme ils voulaient. Des fois ça s'arrangeait, alors ils laissaient tomber une petite pièce dans sa tire-lire, mais des fois aussi ça ne s'arrangeait pas, alors ils ne lui donnaient pas un liard et ils le mettaient en pénitence ... (G. Chepfer, Saynètes, paysanneries 2, 1945)

Avec parfois et quelquefois, la corrélation peut avoir trois maillons, contenant deux contraires, P1 et P2, et un argument indépendant, P3 :

[26] Christian est toujours speed, parfois il dit bonsoir, parfois non, parfois son nez est blanc de poudre, de cocaïne pas bien reniflée. (D. Belloc, Képas, 1989)

Au-delà de trois maillons, les éléments sont regroupés par deux ou par trois et décrivent plusieurs facettes d'un même ensemble.

Les connecteurs, lorsqu'ils sont utilisés, soulignent l'opposition (et est utilisé plutôt que mais ou au contraire). Si, utilisé dans les corrélations avec parfois ou quelquefois, n'introduit pas une hypothèse, mais un point de vue, et l'argument contraire est présenté dans la proposition principale. La force des assertions confrontées est atténuée avec parfois ou quelquefois :

[27] Si les réalistes voient parfois le paysan plus grossier qu'il ne l'est réellement, il est certain que les idéalistes l'ont parfois quintessencié. (G. Sand, Promenades autour d'un village, 1866)

\begin{tabular}{|c|c|c|c|}
\hline & Parfois & Quelquefois & Des fois \\
\hline \multicolumn{4}{|c|}{ Volumes concernés par les relations d'addition } \\
\hline Total des relations d'addition & 58 & 49 & 22 \\
\hline Relations d'addition renforcées par des connecteurs & 10 & 18 & \\
\hline Relations d'addition clôturées par des connecteurs & 8 & 8 & 4 \\
\hline \multicolumn{4}{|c|}{ Nombre de maillons des relations d'addition } \\
\hline Deux maillons & 28 & 29 & 13 \\
\hline Trois maillons & 23 & 13 & 7 \\
\hline Quatre maillons & 3 & 5 & 1 \\
\hline Cinq maillons & 4 & 1 & \\
\hline Six maillons & & & 1 \\
\hline Huit maillons & & 1 & \\
\hline \multicolumn{4}{|c|}{ Nature des connecteurs renforçant les relations d'addition } \\
\hline Aussi & 2 & 3 & \\
\hline Et & 5 & 17 & 3 \\
\hline Et ... aussi & 1 & & \\
\hline Et ... encore & 1 & & \\
\hline Et ... même & 1 & & \\
\hline Encore & 3 & & \\
\hline Même & 2 & 1 & \\
\hline Mais & & 2 & \\
\hline Mais ... aussi & 1 & & \\
\hline Non seulement ... mais ... voire & & 1 & \\
\hline Ou, ou bien & 2 & 1 & \\
\hline Ou ....aussi & & 1 & \\
\hline Puis & & & 1 \\
\hline
\end{tabular}

Tableau 6 : caractéristiques des relations d'addition 
68

Dans les relations d'addition, les segments corrélés constituent une liste d'éléments complémentaires, non contradictoires. Le tableau 6 présente les caractéristiques de ces relations. La corrélation ne couvre pas toujours toutes les possibilités de réalisation :

[28] C'est son nid. Des fois, il fait gonfler son beau ventre tout écaillé d'écume ; des fois il s'étire entre deux os aigus de la roche ; des fois il fait nuit tout à fait et alors on voit seulement son gros oeil couleur d'herbe qui clignote et qui guette. (J. Giono, Regain, 1930)

69 accentuer l'effet de liste :

[29] Et puis suivait l'impossibilité d'avancer encore, ou le sommeil, ou quelquefois rien, ou quelquefois au contraire les achats de maisons, les déménagements, ou quelquefois aussi cette humeur-là, seulement cette humeur, cet accablement ou quelquefois, une reine, tout ce qu'on lui demandait, tout ce qu'on lui offrait, cette maison sur le Petit Lac, sans raison aucune, mon père déjà mourant, ou ce chapeau à bords plats, parce que la petite le voulait tant, ou ces chaussures lamé or idem. Ou rien, ou dormir, mourir. (M. Duras, L'Amant, 1984)

\begin{tabular}{|c|c|c|c|}
\hline & Parfois & Quelquefois & Des fois \\
\hline \multicolumn{4}{|c|}{ Volumes concernés par les relations d'imbrication } \\
\hline Total des relations d'imbrication & 2 & 10 & 3 \\
\hline Relations d'imbrication renforcées par des connecteurs & & 4 & 1 \\
\hline \multicolumn{4}{|c|}{ Nombre de maillons des relations d'imbrication renforcées par des connecteurs } \\
\hline Deux maillons & 1 & 10 & 3 \\
\hline \multicolumn{4}{|l|}{ Trois maillons } \\
\hline Quatre maillons & 1 & & \\
\hline \multicolumn{4}{|l|}{ Six maillons } \\
\hline \multicolumn{4}{|l|}{ Huit maillons } \\
\hline \multicolumn{4}{|c|}{ Nature des connecteurs renforçant les relations d'imbrication } \\
\hline Et & & 2 & \\
\hline Même & & 1 & \\
\hline Si, et si & & 1 & 1 \\
\hline
\end{tabular}

Tableau 7 : caractéristiques des relations d'imbrication

Dans les relations d'imbrication, les occasions dénotées par le premier segment servent de cadre aux possibilités de réalisation du suivant. Le tableau 7 présente les caractéristiques de ces relations. Dans l'exemple [30], le premier élément concerne les «voix qui parfois montaient vers eux», et les autres éléments proposent trois qualifications alternatives de ces voix. L'imbrication s'opère donc sur deux ensembles, le premier segment d'une part, les trois autres d'autre part. L'attente du lecteur, après le premier parfois, est trompée, et le texte nécessite une réanalyse à la lecture du deuxième parfois :

[30] [...] sourds [les deux officiers] aux voix qui parfois montaient vers eux des bords de la route, parfois geignardes, parfois suppliantes, parfois alarmées (...) (C. Simon, L'Acacia, 1989) 
Les corrélations avec quelquefois et des fois présentent des schémas où chaque segment corrélé est introduit par si et contient la proposition hypothétique et la principale; le second segment s'inscrit dans l'hypothèse de réalisation du premier :

[31] Tu es bien loin toi-même, ma noble amie, de soupçonner de qui je veux parler; si le souvenir de ce vil drôle se présente quelquefois à ton esprit, tu l'accueilles sans répugnance, si tu parles quelquefois de lui, c'est avec amitié. (V. Hugo, Lettres à la fiancée, 1822)
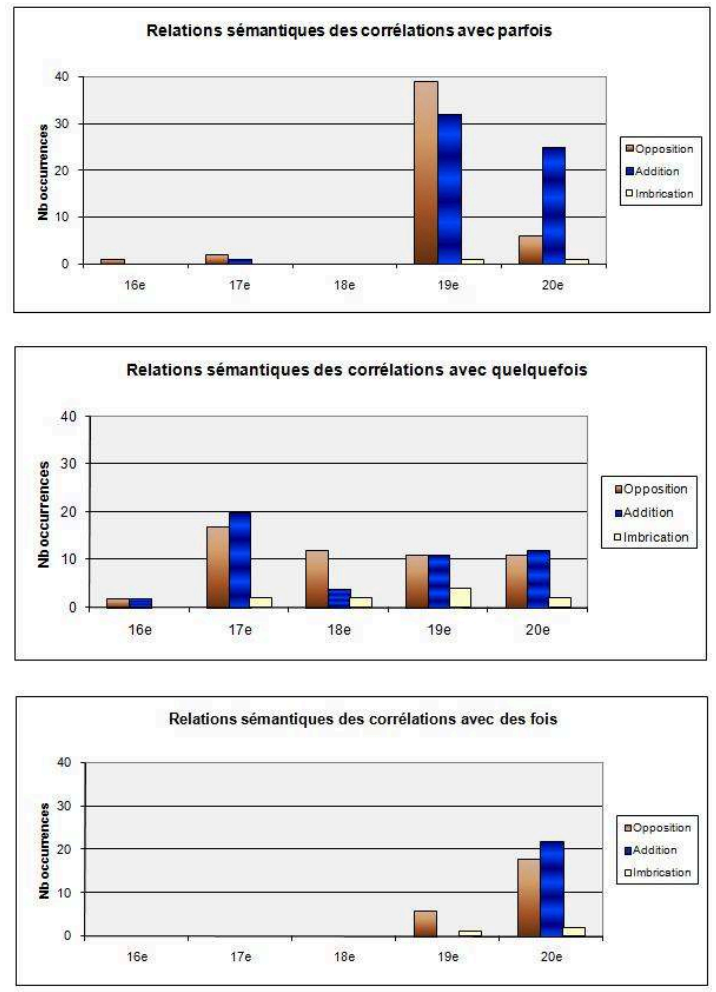

Figure 2 : comparaison par siècle des relations sémantiques dans les corrélations avec parfois, quelquefois, des fois

72 La figure 2 présente les graphiques comparés des relations sémantiques dans les corrélations avec parfois, quelquefois, des fois ventilées chronologiquement. Les corrélations avec parfois tendent à devenir moins oppositives et plus énumératives. Les relations d'opposition avec des fois restent nombreuses, rappelant l'aptitude de des fois à la controverse.

73 Nous avons analysé la portée des corrélations et recherché s'il était fréquent qu'elles s'étendent au-delà de la phrase graphique ${ }^{7}$. Le plus souvent, la portée est limitée à la phrase graphique. $\mathrm{Au} \mathrm{xx}^{\mathrm{e}}$ siècle, quelques corrélations avec parfois et de nombreuses corrélations avec des fois ${ }^{8}$ portent au-delà. La forme est placée en tête de segment, elle peut être précédée d'un connecteur ou d'une interjection :

[32] Parfois le vice-consul parle très longtemps de façon inintelligible. Parfois son discours est clair. (M. Duras, Le Vice-Consul, 1965)

[33] Oui, Saturnin des fois, il pense comme ça. Mais d'autres fois, le concierge Saturnin il est pas du tout comme ça. (R. Queneau, Le Chiendent, 1933) 


\subsubsection{Aux frontières de la corrélation : zone d'antécédence et zone post- corrélative}

74 Aux marges gauche et droite de la zone de corrélation, des marqueurs relient la corrélation au reste du texte. Le tableau 8 présente la répartition par siècle des corrélations introduites et fermées par des marqueurs.

\begin{tabular}{|l|c|c|c|c|c|c|}
\cline { 2 - 6 } \multicolumn{1}{c|}{} & $\begin{array}{c}\text { Nb corrélations ayant des marqueurs } \\
\text { avant } \\
\text { parfois }\end{array}$ & $\begin{array}{c}\text { avant et } \\
\text { après } \\
\text { corrélation } \\
\text { corrélation }\end{array}$ & $\begin{array}{c}\text { Noprès } \\
\text { corrélation }\end{array}$ & $\begin{array}{c}\text { Nb corrélations } \\
\text { marquées }\end{array}$ & $\begin{array}{c}\text { Nb total de } \\
\text { corrélations }\end{array}$ & $\%$ \\
\hline $16 \mathrm{e}$ siècle & 1 & 1 & 1 & 1 & $100 \%$ \\
$17 \mathrm{e}$ siècle & 1 & 1 & 6 & 8 & 3 & $33 \%$ \\
$19 \mathrm{e}$ siècle & 2 & 2 & 4 & 32 & $11 \%$ \\
$20 \mathrm{e}$ siècle & 1 & 4 & 9 & 14 & 108 & $13 \%$ \\
\hline \multicolumn{1}{|c|}{ Total parfois } & & & & & & $13 \%$ \\
\hline
\end{tabular}

\begin{tabular}{|l|l|l|l|l|l|l|}
\hline quelquefois & \multicolumn{2}{|c|}{} & \multicolumn{3}{|l|}{} \\
\hline 16 e siècle & 1 & & 1 & 2 & 4 & $50 \%$ \\
17 e siècle & 2 & 3 & 10 & 15 & 39 & $38 \%$ \\
$18 \mathrm{e}$ siècle & 1 & 3 & 4 & 8 & 18 & $44 \%$ \\
$19 \mathrm{e}$ siècle & & & 6 & 6 & 26 & $23 \%$ \\
$20 \mathrm{e}$ siècle & 1 & & 6 & 7 & 25 & $28 \%$ \\
\hline Total quelquefois & 5 & 6 & 27 & 38 & 112 & $34 \%$ \\
\hline
\end{tabular}

\begin{tabular}{|l|l|l|l|l|l|l|}
\hline \multicolumn{1}{|c|}{ des fois } & \multicolumn{2}{|c|}{} & 1 & 1 & 7 & $14 \%$ \\
\hline $19 e$ siècle & & & 6 & 9 & 42 & $21 \%$ \\
\hline 20 e siècle & 2 & 1 & 7 & 10 & 49 & $20 \%$ \\
\hline Total des fois & 2 & 1 & 7 &
\end{tabular}

Tableau 8 : répartition par siècle des corrélations avec parfois, quelquefois, des fois contenant des connecteurs extérieurs à la ZC

75 Le nombre de corrélations concernées est faible (13 à 34\%). La partie droite de la corrélation semble plus balisée.

A gauche de la corrélation, les marqueurs sont variés et précèdent immédiatement la zone de corrélation. Ils annoncent que le texte passe à une nouvelle phase. Dans les corrélations avec des fois, des interjections (alors, eh bien) introduisent la corrélation en réponse à une question :

[34] Et alors?

- Alors, des fois il ne répond pas, il soupire, des fois il tremble. Et d'autres fois... grands dieux ! (G. Bernanos, Monsieur Ouine, 1943)

Lorsque des marqueurs bornent la corrélation à gauche et à droite, ils produisent un parallélisme de niveau supérieur (c'est que ... et que; car puisque ... et qu'après). Les marqueurs de gauche peuvent se situer plus en amont de la corrélation, mais les marqueurs finaux suivent de près les segments corrélés. Les constructions dépassent les limites de la phrase et assurent la continuité discursive du texte. Le parallélisme le plus marqué entre la borne de début et la borne de fin se trouve dans les occurrences avec parfois et des fois au $\mathrm{xx}^{\mathrm{e}}$ siècle : [35] et [37]. Les exemples avec quelquefois, comme [36], sont plus hétérogènes et plus anciens : 
[35] Cette lettre, du reste, l'accusation, tout en la relevant, n'en tient pas grand compte. Il arrive parfois, souvent même, que le procureur reçoive de la prison semblables " aveux " destinés parfois à éclairer la justice, parfois à l'égarer ; lettres écrites, parfois même, sans but et sans raison, dans le désoeuvrement de la geôle. (A. Gide, Souvenirs de la Cour d'assises, 1913)

[36] Tantôt c'étaient des promenades où les beautés de la cour, à cheval, faisaient assaut de grâces et d'attraits : quelquefois bien, quelquefois mal, mais toujours de leur mieux. D'autres fois on voyait sur la rivière un spectacle que la seule ville de Londres peut offrir. (A. Hamilton, Mémoires de la vie du comte de Gramont, 1713)

[37] Mais à présent ils perdent tout le temps. Dans le temps, il y avait des métiers, ils avaient chacun son métier; et dans les métiers des fois ils servaient à perdre, mais des fois ils servaient à gagner. Et à présent on perd tout le temps. (C. Péguy, Le Mystère de la charité de Jeanne d'Arc, 1910)

Les connecteurs post-corrélatifs sont les plus répandus. Ils assurent la transition avec la suite du texte. Les plus fréquents sont des coordonnants. D'autres apportent une conclusion : c'est pourquoi, et ceci, ainsi, enfin :

[38] Il résulta de cet amalgame (plus vraisemblable au fond qu'on ne croirait) un drame tout à fait bizarre, parfois sublime et parfois ridicule, jamais odieux ; et ceci est le plaisant de l'affaire. (G. Sand, Histoire de ma vie, 1855)

Avec parfois ou quelquefois, des adverbes de temps : à tout moment, sans cesse, opposent la permanence d'une situation ou d'une action aux alternances décrites dans les segments corrélés. D'autres adverbes de temps : soudain, alors, tout de suite, marquent une rupture dans le récit.

Le marqueur post-corrélatif reste proche de la zone de corrélation, sauf dans un cas, où il en est séparé par une phrase complète. La corrélation est constituée de trois maillons avec parfois, la zone post-corrélative reprend parfois dans une phrase nouvelle, et souligne la clôture par quand même :

[39] Il n'en avait pas moins une extraordinaire passion de nouvelles. En entendant sonner à la grille, il courait, songeant au facteur. C'était parfois un commerçant, parfois le boulanger, parfois la bouchère, avec sa rosse et sa chignolle. Jusserand revenait furieux. Parfois c'était quand même le facteur. (G. Duhamel, Chronique des Pasquier, 1937)

De cette analyse, il ressort que :

- Parfois n'apparaît régulièrement en corrélation qu'à partir du XIX siècle; la corrélation exprime d'abord l'opposition, puis l'addition. Sa portée est majoritairement limitée à la phrase graphique. L'usage de si en corrélation : si parfois P1, parfois P2 oppose deux points de vue, alors que si parfois en forme simple perd son sens temporel au profit d'une valeur hypothétique et modale. En emploi simple et corrélé, parfois permet de modérer le propos énoncé dans la proposition en si.

- Quelquefois est la forme la plus ancienne et la plus variée. Sa portée est limitée à la phrase graphique. Son emploi en corrélation est peut-être en déclin. L'usage de si en corrélation : $s i$ quelquefois P1, quelquefois P2 oppose deux arguments. L'emploi hypothétique reste possible dans les relations d'imbrication, où chaque segment corrélé contient à la fois la proposition subordonnée et la proposition principale. En emploi simple et corrélé, quelquefois atténue le propos énoncé dans la proposition en si. 
- Des fois est la forme la plus récente et celle qui utilise le moins de connecteurs; elle porte fréquemment au-delà de la phrase. Elle exprime plus l'opposition que l'addition. A partir d'un seul élément, elle peut sous-entendre l'élément complémentaire. Des fois s'associe à d'autres fois de façon privilégiée, et peut se corréler avec des repères d'énonciation (ici, maintenant). La corrélation avec si des fois en emploi hypothétique est possible dans les relations d'imbrication. Comme en emploi simple, la présence de des fois réduit les possibilités de réalisation de la proposition en si.

\section{Conclusion : comparaison des emplois de parfois, quelquefois, des fois}

82 La comparaison entre les trois formes en emploi simple paraît isoler, d'un côté parfois et quelquefois, de l'autre des fois; en effet, des fois est plus familier, d'apparition récente, et plus souvent extraprédicatif. En corrélation, il parait plus juste de distinguer quelquefois d'une part, et parfois et des fois d'autre part ; les corrélations avec quelquefois sont plus hétérogènes et plus anciennes; parfois et des fois en corrélation se placent souvent en tête de segment et facilitent ainsi le parallélisme de la construction d'ensemble; les corrélations bornées à droite et à gauche par des marqueurs élargissent l'unité textuelle au-delà de la phrase. La différence entre parfois et des fois en français moderne apparaît ailleurs : dans le registre de langue, dans la préférence de parfois à l'écrit ou de des fois à l'oral, et dans la tendance à dépasser les limites de la phrase, plus nette avec des fois.

Nous avons situé les corrélations avec parfois, quelquefois, des fois au bas de l'échelle de dépendance entre les éléments phrastiques. A la différence des corrélateurs " forts », le premier segment n'implique pas la présence d'un second. Plutôt que d'emploi corrélatif, il faudrait parler d'emploi en corrélation.

L'étude du corpus nous a permis de détailler certains mécanismes par lesquels s'établissent les rapprochements entre les segments. Lorsque la structure interne du segment est modifiée (antéposition de la forme parfois, quelquefois ou des fois, répétition du connecteur en tête de chaque segment), la corrélation se prête bien à des phénomènes de série à plusieurs maillons, ou passe facilement au-delà de la phrase graphique. Au niveau lexical, les formes parfois, quelquefois, des fois forment des combinaisons entre elles et avec d'autres adverbes temporels, et constituent des sousgroupes de segments à l'intérieur de la corrélation, représentant plusieurs facettes d'une situation. Enfin, nous avons relevé des formes complexes de corrélation, qui nécessitent souvent une réanalyse de la part du lecteur (relations d'imbrication, corrélations bornées à gauche et à droite par des marqueurs). Ces structures sont souvent récentes, peu fréquentes, mais elles montrent que les relations entre les segments restent perceptibles dans un schéma de construction sophistiqué. 


\section{BIBLIOGRAPHIE}

ALLAIRE, S. 1997. Le modèle syntaxique des systèmes corrélatifs. Thèse de Doctorat d'Etat : Université

Rennes 2.

ATILF, base textuelle Frantext [Consultation du 01/09/2006 au 28/02/2008] via le portail Virtuose des bibliothèques de la Sorbonne nouvelle. http://www.scd.univ-paris3.fr

ATILF, TLF Le Trésor de la Langue Française informatisé [Consultation du 27/10/2006 au 28/03/2008]. http://atilf.atilf.fr

BERTHONNEAU, A.-M. 1989. Composantes linguistiques de la référence temporelle. Les compléments de temps, du lexique à l'énoncé. Thèse de doctorat d'Etat : Université Paris VII.

CHAROLLES, M. 2001. De la phrase au discours : quelles relations ? La sémantique des relations. A. ROUSSEAU (éd). Université Lille3, coll. Travaux et Recherches. 236-260.

DESWART, H. 1998. Quelque fois and quelquefois : about iteration and frequency in French. Linguistics in the Netherlands. 149-158

GAFFIOT, F., FLOBERT, P. 2001. Dictionnaire Latin-Français. Paris : Hachette.

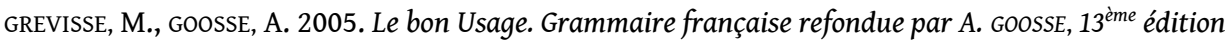
revue. Bruxelles : Duculot.

GUIMIER, C. 1996. Les adverbes du français. Le cas des adverbes en -ment. Paris : Ophrys.

KERBRAT-ORECCHIONI, C. 2005. Le discours en interaction. Paris : A. Colin.

REY, A., TOMI, M., TANET, C. 1999. Dictionnaire historique de la langue française. Paris : Dictionnaire Le Robert.

SCHNEDECKER, C. (2006). De l'un à l'autre et réciproquement. Aspects sémantiques, discursifs et cognitifs des pronoms anaphoriques corrélés (l'un/l'autre et le premier/le second). Bruxelles : De Boeck

STUMP, G. 1981. The interpretation of frequency adverbs. Linguistics and Philosophy $4: 221-227$

TRAUGOTT, E. 1995. Subjectification in grammaticalization. Subjectivity and subjectivisation. Linguistic perspectives. D. STEIN, S. WRIGHT (éds). Cambridge : Cambridge University Press. 31-54.

TRAUGOTT, E. 2003. From Subjectification to intersubjectification. Motive for Language Change. Cambridge : Cambridge University Press. 124-139.

\section{NOTES}

1. L'appréciation de l'événement considéré transparaît dans le langage par l'ajout d'un adjectif qualificatif ou d'un adverbe : une bonne fois, une fois de trop,...

2. Le Trésor de la Langue Française Informatisé.

3. Cette dernière précision nous a été communiquée par B. Fagard, CR au Cnrs (UMR 8094 LatticeEns)

4. Frantext regroupe des textes de 1507 à 2006, auxquels sont adjoints deux textes des XIIe et XIIIle siècles

Recherche des constructions où parfois, quelquefois, des fois apparaissent au moins deux fois. Complément de recherche pour des fois : constructions contenant des fois et d'autres fois. Il est à 
noter que, dans le cas de corrélations étendues, Frantext retourne toutes les occurrences correspondant à la requête formulée ; ce mode de recherche fournit en plusieurs occurrences un extrait composé de plus de deux exemplaires des formes recherchées, qu'il faut recomposer lors du dépouillement.

Recherche dans Frantext d'extraits contenant parfois en un seul exemplaire combiné à quelquefois ou des fois ; puis d'extraits contenant quelquefois en un seul exemplaire combiné à parfois ou des fois ; enfin, d'extraits contenant des fois en un seul exemplaire combiné à parfois ou quelquefois.

Recherche de parfois, quelquefois ou des fois seuls, mais suivis d'adverbes de fréquence: jamais, toujours, rarement, souvent.

Recherche de des fois seul, non suivi de d'autres fois.

5. 1026 résultats pour parfois, 482 pour quelquefois

6. Pour nos statistiques, nous avons choisi arbitrairement de comptabiliser cette occurrence dans les corrélations avec quelquefois

7. Recherche des ponctuations fortes (point) entre les maillons de la corrélation

8. $3 \%$ des corrélations avec parfois, $40 \%$ des corrélations avec des fois

\section{RÉSUMÉS}

Nous étudions l'emploi corrélatif des formes parfois, quelquefois, des fois et leur aptitude à délimiter un segment de discours. Après avoir identifié les propriétés sémantiques et syntaxiques de ces formes en emploi simple, nous définissons les caractéristiques de la corrélation qui peuvent être prises en compte avec parfois, quelquefois, des fois. Nous exploitons un corpus du XVI au $\mathrm{xx}^{\mathrm{e}}$ siècle constitué à partir de Frantext, contenant des énoncés conformes à nos critères, et mettons en valeur les propriétés sémantiques et syntaxiques de ces formes en emploi corrélatif. Nous comparons ensuite les propriétés de parfois, quelquefois, des fois en emploi simple et en corrélation pour mettre en valeur les différences.

We will study the correlative uses of the forms parfois (sometimes), quelquefois (now and then), des fois (several times) and how they mark the boundaries of a segment of discourse. After having identified the semantic and syntactical properties of these forms when used on their own, we will then define the characteristics of the correlations established with parfois, quelquefois and des fois. We will draw on a corpus from the $16^{\text {th }}$ through the $20^{\text {th }}$ century from the FRANTEXT online library, including utterances which we expect will meet our criteria and we will discuss the semantic and syntactical characteristics of these forms in correlative uses. We will finally compare the properties of parfois, quelquefois, des fois used on their own and in their correlative uses in order to highlight their differences.

\section{INDEX}

Mots-clés : zone de corrélation, unité textuelle, cohérence du discours

Keywords : correlation area, textual unity, discourse coherence 
AUTEUR

ANNE-MARIE GUIRAUD

UMR LATTICE, Université de Paris III anne-marie.guiraud@dbmail.com 